\title{
Finite Element Model of Functionally Graded Nanobeam for Free Vibration Analysis
}

\author{
Büşra Uzun ${ }^{a *}$, Mustafa Özgür Yaylı ${ }^{b}$ \\ ${ }^{a, b}$ Bursa Uludag University, Civil Engineering Department \\ Division of Mechanics, Bursa-TURKIYE \\ ${ }^{*}$ E-mail address: buzun@uludag.edu.tr ${ }^{\mathrm{a}^{*}}, \underline{\text { ozguryayli@uludag.edu.tr }}{ }^{\text {b }}$ \\ ORCID numbers of authors:

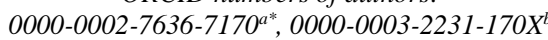

Received date: 24.05 .2019

Accepted date: 12.06 .2019

\begin{abstract}
In the present study, free vibration of functionally graded (FG) nanobeam is investigated. The variation of material properties is assumed in the thickness direction according to the power law. FG nanobeam is modeled as Euler-Bernoulli beam with different boundary conditions and investigated based on Eringen's nonlocal elasticity theory. Governing equations are derived via Hamilton principle. Frequency values are found by using finite element method. FG nanobeam is composed of silicon carbide (SiC) and stainless steel (SUS304). The effects of dimensionless small-scale parameters $\left(e_{0} a / L\right)$, power law exponent $(k)$ and boundary conditions on frequencies are examined for FG nanobeam.
\end{abstract}

Keywords: Functionally graded nanobeam, nonlocal elasticity theory, free vibration, finite element method

\section{Introduction}

Functionally graded materials (FGMs) are defined as special composites which material properties change continuously along with direction of the material. FGMs are mostly composed of ceramic and metal. Thus the ceramic can resist high temperature in thermal environments, while the metal can reduce the stress occurring on the ceramic surface at the earlier case of cooling. FGMs are utilized in various applications such as aviation, mechanical, electronics, nuclear, optics, chemical, biomedicine and civil engineering [1-2].

The classical continuum theories lose their validity when the dimensions are reduced because they lack internal/additional material small-scale parameters. For this reason, some researchers have been used some higher order theories that take into account small-scale effect analysis of micro and nano structures [3-5]. Among higher order theories, nonlocal elasticity theory [6] have been widely studied recently [7-21]. Ebrahimi et al. [2] presented the applicability of differential transformation method (DTM) in investigations on vibrational characteristics of FG size-dependent nanobeams. Civalek and Demir [22] developed elastic beam model using nonlocal elasticity theory and Euler-Bernoulli beam theory for the bending 
analysis of microtubules (MTs). Kadığlu and Yaylı [23] studied buckling analysis of a nano sized beam by using Timoshenko beam theory and Eringen's nonlocal elasticity theory. Zargaripoor et al. [24] investigated free vibration of functionally graded nanoplate by using Eringen's nonlocal theory.

In this study, vibration characteristics of FG nanobeams are investigated. The variation of material properties is assumed in the thickness direction based on the power law. FG nanobeam is composed of silicon carbide ( $\mathrm{SiC}$ ) and stainless steel (SUS304). Governing equations are derived via Hamilton principle. The vibration behaviours of SiC/SUS304 FG nanobeam with simply-supported (S-S) and clamped-clamped (C-C) boundary conditions are analyzed using nonlocal finite element formulation. The effects of small-scale parameters $\left(e_{0} / L\right)$, power law exponents $(k)$ and boundary conditions on frequencies are examined for FG nanobeam.

\section{Functionally Graded Euler-Bernoulli Beam}
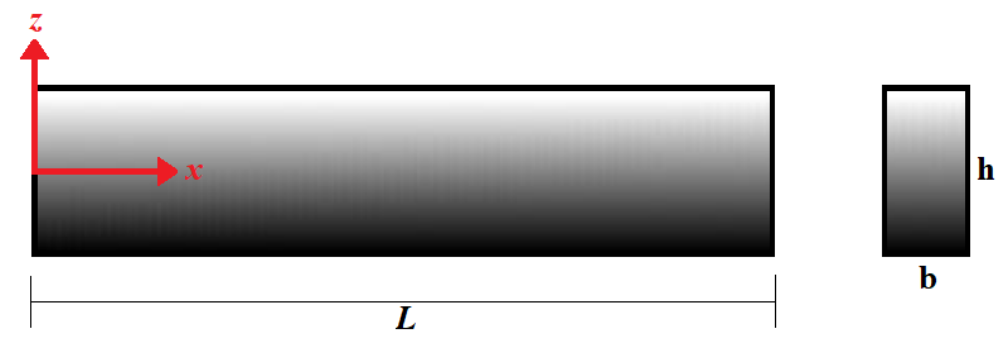

Fig. 1. Ilustration of FG beam

$L, b$ and $h$ are length, width and thickness of the FG beam, respectively. The material properties of the beam are assumed to vary continuously in the thickness direction. The effective material property of FG beam is expressed by the power law as follows [9]

$$
P(z)=\left(P_{U}-P_{L}\right)\left(\frac{z}{h}+\frac{1}{2}\right)^{k}+P_{L}
$$

Here $P(z)$ is the effective material property of the beam, $P_{U}$ and $P_{L}$ are the material property at the upper and lower surfaces of the beam, $k$ is the power law exponent (non-negative variable parameter). $P(z)$ indicates to the properties of the beam components such as the elastic module $(E)$, density $(\rho)$ etc. and can be transformed into the following forms

$$
E(z)=\left(E_{U}-E_{L}\right)\left(\frac{z}{h}+\frac{1}{2}\right)^{k}+E_{L}
$$




$$
\rho(z)=\left(\rho_{U}-\rho_{L}\right)\left(\frac{z}{h}+\frac{1}{2}\right)^{k}+\rho_{L}
$$
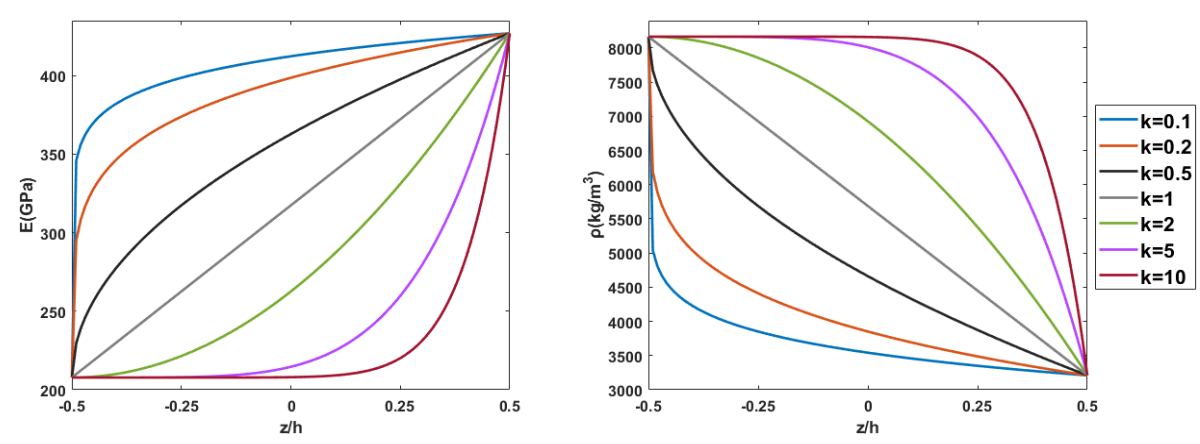

Fig. 2. The variation of material properties through the thickness direction

The displacements for Euler-Bernoulli beam can be written as follows [13]

$$
\begin{gathered}
u_{1}(x, z, t)=u(x, t)-z \frac{\partial w(x, t)}{\partial x} \\
u_{2}(x, z, t)=0 \\
u_{3}(x, z, t)=w(x, t)
\end{gathered}
$$

Here $u_{1}, u_{2}$ and $u_{3}$ are the displacements in the $x, y, z$ directions, respectively. $u$ and $w$ denote longitudinal and transverse displacements of any point on the neutral axis, respectively. Strains of the Euler-Bernoulli beam as follows

$$
\varepsilon_{x x}=\frac{\partial u(x, t)}{\partial x}-z \frac{\partial^{2} w(x, t)}{\partial x^{2}}, \varepsilon_{x y}=\varepsilon_{y x}=\varepsilon_{x z}=\varepsilon_{z x}=\varepsilon_{y y}=\varepsilon_{y z}=\varepsilon_{z y}=\varepsilon_{z z}=0
$$

$\varepsilon_{\mathrm{xx}}$ is the non-zero only strain component. Stress, normal force and moment expressions for the functionally graded beam are written as follows

$$
\begin{gathered}
\sigma_{x x}=E(z) \varepsilon_{x x} \\
N=A_{1} \frac{\partial u}{\partial x}-B_{1} \frac{\partial^{2} w}{\partial x^{2}}, M=B_{1} \frac{\partial u}{\partial x}-D_{1} \frac{\partial^{2} w}{\partial x^{2}}
\end{gathered}
$$

$A_{1}, B_{1}$ ve $D_{1}$ are expressed as 


$$
A_{1}=\int_{A} E(z) d A, B_{1}=\int_{A} E(z) z d A, D_{1}=\int_{A} E(z) z^{2} d A
$$

The Hamilton principle to be used to obtain equations of motion is expressed as follows [25]

$$
\int_{0}^{T}(\delta S-\delta T) d t=0
$$

Where $S$ and $T$ are the strain energy and kinetic energy, respectively. $S$ and $T$ for an element which has volume $V$ and length $L$ is as below

$$
\begin{gathered}
S=\frac{1}{2} \int_{V} \sigma_{x x} \varepsilon_{x x} d V \\
T=\frac{1}{2} \int_{V} \rho(z)\left(\left(\frac{\partial u_{1}}{\partial t}\right)^{2}+\left(\frac{\partial u_{2}}{\partial t}\right)^{2}+\left(\frac{\partial u_{3}}{\partial t}\right)^{2}\right) d V
\end{gathered}
$$

The first variation of the strain and kinetic energy are obtained as follows

$$
\begin{gathered}
\delta \int_{0}^{T} S d t=\int_{0}^{T} \int_{0}^{L}\left(N \delta\left(\frac{\partial u}{\partial x}\right)-M \delta\left(\frac{\partial^{2} w}{\partial x^{2}}\right)\right) d x d t \\
=\int_{0}^{T} \int_{0}^{L}\left(\left(A_{1} \frac{\partial u}{\partial x}-B_{1} \frac{\partial^{2} w}{\partial x^{2}}\right) \delta\left(\frac{\partial u}{\partial x}\right)-\left(B_{1} \frac{\partial u}{\partial x}-D_{1} \frac{\partial^{2} w}{\partial x^{2}}\right) \delta\left(\frac{\partial^{2} w}{\partial x^{2}}\right)\right) d x d t \\
\delta \int_{0}^{T} T d t=\int_{0}^{T} \int_{0}^{L}\left(I_{0}\left(\frac{\partial u}{\partial t} \delta\left(\frac{\partial u}{\partial t}\right)+\frac{\partial w}{\partial t} \delta\left(\frac{\partial w}{\partial t}\right)\right)-I_{1}\left(\frac{\partial u}{\partial t} \delta\left(\frac{\partial^{2} w}{\partial x \partial t}\right)+\frac{\partial^{2} w}{\partial x \partial t} \delta\left(\frac{\partial u}{\partial t}\right)\right)+I_{2} \frac{\partial^{2} w}{\partial x \partial t} \delta\left(\frac{\partial^{2} w}{\partial x \partial t}\right)\right) d x d t
\end{gathered}
$$

Here $I_{0}, I_{1}$ and $I_{2}$ are expressed as 


$$
I_{0}=\int_{A} \rho(z) d A, I_{1}=\int_{A} \rho(z) z d A, I_{2}=\int_{A} \rho(z) z^{2} d A
$$

Substituting Equations (12) and (13) into Equation (9), we obtain the equilibrium equations from the Euler-Lagrange equation as follows

$$
\begin{gathered}
\delta u: \frac{\partial N}{\partial x}=I_{0} \frac{\partial^{2} u}{\partial t^{2}}-I_{1} \frac{\partial^{3} w}{\partial x \partial t^{2}} \\
\delta w: \frac{\partial^{2} M}{\partial x^{2}}=I_{0} \frac{\partial^{2} w}{\partial t^{2}}+I_{1} \frac{\partial^{3} u}{\partial x \partial t^{2}}-I_{2} \frac{\partial^{4} w}{\partial x^{2} \partial t^{2}}
\end{gathered}
$$

\section{Nonlocal Functionally Graded Nanobeam}

The nonlocal constitutive formulation is [6]

$$
\left[1-\left(e_{0} a\right)^{2} \nabla^{2}\right] \sigma_{i j}=C_{i j k l} \varepsilon_{k l}
$$

Where $\sigma_{i j}$ is the stress tensor, $C_{i j k l}$ is the fourth-order elastic module tensor, $\varepsilon_{k l}$ is the strain tensor, $e_{0}$ is a material constant which is determined experimentally, $a$ is the internal characteristic length. For Euler-Bernoulli FG nanobeam, Equation (17) can be rewritten as

$$
\sigma_{x x}-\left(e_{0} a\right)^{2} \frac{\partial^{2} \sigma_{x x}}{\partial x^{2}}=E(z) \varepsilon_{x x}
$$

Integrating Equation (18) over the cross-section area, we obtain the axial force-strain relation as

$$
N-\left(e_{0} a\right)^{2} \frac{\partial^{2} N}{\partial x^{2}}=A_{1} \frac{\partial u}{\partial x}-B_{1} \frac{\partial^{2} w}{\partial x^{2}}
$$

Multiplying Equation (18) by $\mathrm{z}$ and integrating over the cross-section area, we get the moment-curvature relation as 


$$
M-\left(e_{0} a\right)^{2} \frac{\partial^{2} M}{\partial x^{2}}=B_{1} \frac{\partial u}{\partial x}-D_{1} \frac{\partial^{2} w}{\partial x^{2}}
$$

Differentiating Equation (15) with respect to $\mathrm{x}$, then substituting Equation (19) we obtain Equation (21). And substituting Equation (16), we obtain Equation (22).

$$
\begin{gathered}
N=A_{1} \frac{\partial u}{\partial x}-B_{1} \frac{\partial^{2} w}{\partial x^{2}}+\left(e_{0} a\right)^{2}\left(I_{0} \frac{\partial^{3} u}{\partial x \partial t^{2}}-I_{1} \frac{\partial^{4} w}{\partial x^{2} \partial t^{2}}\right) \\
M=B_{1} \frac{\partial u}{\partial x}-D_{1} \frac{\partial^{2} w}{\partial x^{2}}+\left(e_{0} a\right)^{2}\left(I_{0} \frac{\partial^{2} w}{\partial t^{2}}+I_{1} \frac{\partial^{3} u}{\partial x \partial t^{2}}-I_{2} \frac{\partial^{4} w}{\partial x^{2} \partial t^{2}}\right)
\end{gathered}
$$

\section{Finite Element Formulation}

The variational statement of FG Euler-Bernoulli nanobeam has the following form

$$
\left.\int_{0}^{T} \int_{0}^{L}\left(\begin{array}{l}
\left(\begin{array}{l}
A_{1} \frac{\partial u}{\partial x} \delta\left(\frac{\partial u}{\partial x}\right)-B_{1} \frac{\partial^{2} w}{\partial x^{2}} \delta\left(\frac{\partial u}{\partial x} \delta\left(\frac{\partial^{2} w}{\partial x^{2}}\right)-D_{1} \frac{\partial^{2} w}{\partial x^{2}} \delta\left(\frac{\partial^{2} w}{\partial x^{2}}\right)+\left(e_{0} a\right)^{2}\left(I_{0} \frac{\partial^{3} u}{\partial x \partial t^{2}} \delta\left(\frac{\partial u}{\partial x}\right)-I_{1} \frac{\partial^{4} w}{\partial x^{2} \partial t^{2}} \delta\left(\frac{\partial u}{\partial x}\right)\right)\right) \\
+\left(e_{0} a\right)^{2}\left(I_{0} \frac{\partial^{2} w}{\partial t^{2}} \delta\left(\frac{\partial^{2} w}{\partial x^{2}}\right)+I_{1} \frac{\partial^{3} u}{\partial x \partial t^{2}} \delta\left(\frac{\partial^{2} w}{\partial x^{2}}\right)-I_{2} \frac{\partial^{4} w}{\partial x^{2} \partial t^{2}} \delta\left(\frac{\partial^{2} w}{\partial x^{2}}\right)\right)
\end{array}\right) \\
-\left(I_{0}\left(\frac{\partial u}{\partial t} \delta\left(\frac{\partial u}{\partial t}\right)+\frac{\partial w}{\partial t} \delta\left(\frac{\partial w}{\partial t}\right)\right)-I_{1}\left(\frac{\partial u}{\partial t} \delta\left(\frac{\partial^{2} w}{\partial x \partial t}\right)+\frac{\partial^{2} w}{\partial x \partial t} \delta\left(\frac{\partial u}{\partial t}\right)\right)+I_{2} \frac{\partial^{2} w}{\partial x \partial t} \delta\left(\frac{\partial^{2} w}{\partial x \partial t}\right)\right)
\end{array}\right)\right) d t
$$

$\phi u$ and $\phi w$ are the interpolation shape functions and they are expressed as below

$$
\begin{gathered}
{\left[\phi_{u}\right]=\left[\begin{array}{ll}
1-\frac{x}{L} & \frac{x}{L}
\end{array}\right]} \\
{\left[\phi_{w}\right]=\left[\begin{array}{llll}
1-\frac{3 x^{2}}{L^{2}}+\frac{2 x^{3}}{L^{3}} & x-\frac{2 x^{2}}{L}+\frac{x^{3}}{L^{2}} & \frac{3 x^{2}}{L^{2}}-\frac{2 x^{3}}{L^{3}} & -\frac{x^{2}}{L}+\frac{x^{3}}{L^{2}}
\end{array}\right]}
\end{gathered}
$$

The stiffness matrices $\left(K_{u}, K_{u w}, K_{w}\right)$, the classical mass matrices $\left(M_{u}^{c}, M_{u w}^{c}, M_{w}^{c}\right)$ and the nonlocal mass matrices ( $M_{u}^{n l}, M_{u w}^{n l}, M_{w}^{n l}$ ) are obtained using Equations (23)-(25) as follows

$$
K_{u}=\int_{0}^{L} A_{1}\left(\left[\phi_{u}\right]^{\prime}\right)^{T}\left[\phi_{u}\right]^{\prime} d x
$$




$$
\begin{gathered}
K_{u w}=-\left(\int_{0}^{L} B_{1}\left(\left[\phi_{w}\right]^{\prime \prime}\right)^{T}\left[\phi_{u}\right]^{\prime} d x+\int_{0}^{L} B_{1}\left(\left[\phi_{u}\right]^{\prime}\right)^{T}\left[\phi_{w}\right]^{\prime \prime} d x\right) \\
K_{w}=\int_{0}^{L} D_{1}\left(\left[\phi_{w}\right]^{\prime \prime}\right)^{T}\left[\phi_{w}\right]^{\prime \prime} d x \\
M_{u}^{c}=\int_{0}^{L} I_{0}\left(\left[\phi_{u}\right]\right)^{T}\left[\phi_{u}\right] d x \\
M_{u w}^{c}=-\left(\int_{0}^{L} I_{1}\left(\left[\phi_{u}\right]\right)^{T}\left[\phi_{w}\right]^{\prime} d x+\int_{0}^{L} I_{1}\left(\left[\phi_{w}\right]^{\prime}\right)^{T}\left[\phi_{u}\right] d x\right) \\
M_{w}^{c}=\int_{0}^{L} I_{0}\left(\left[\phi_{w}\right]\right)^{T}\left[\phi_{w}\right] d x+\int_{0}^{L} I_{2}\left(\left[\phi_{w}\right]^{\prime}\right)^{T}\left[\phi_{w}\right]^{\prime} d x \\
M_{w}^{n l}=-\left(e_{0} a\right)^{2} \int_{0}^{L} I_{0}\left(\left[\phi_{w}\right]\right)^{T} \frac{\partial^{2}\left[\phi_{w}\right]}{\partial x^{2}} d x+\left(e_{0} a\right)^{2} \int_{0}^{L} I_{2}\left(\frac{\partial^{2}\left[\phi_{w}\right]}{\partial x^{2}}\right)^{T} \frac{\partial^{2}\left[\phi_{w}\right]}{\partial x^{2}} d x \\
\left.\left.M_{u}^{n l}=-\left(e_{0} a\right)^{2} \int_{0}^{L} I_{1}\left(\left[\phi_{u}\right]^{\prime}\right)^{T}\left[\phi_{w}\right]^{\prime \prime} d x+\left(e_{0} a\right)^{2} \int_{0}^{L} I_{0}\left(\left[\phi_{1}\right]^{\prime}\right)^{T}\left[\phi_{w}\right]^{\prime} d x\right]^{\prime \prime}\left[\phi_{u}\right]^{\prime} d x\right)
\end{gathered}
$$

The frequencies of FG nanobeam are found as follows

$$
\left|K-\omega^{2} M\right|=0
$$

Here $\omega$ is frequency. $K$ and $M$ are total stiffness and mass matrices and given in Equations (36) and (37)

$$
\begin{gathered}
K=K_{u}+K_{w}+K_{u w} \\
M=M_{u}^{c}+M_{u w}^{c}+M_{w}^{c}+M_{u}^{n l}+M_{u w}^{n l}+M_{w}^{n l}
\end{gathered}
$$




\section{Numerical Results for Free Vibration of FG Nanobeam}

In this section frequency values of SiC/SUS304 FG nanobeam are obtained with various dimensionless small-scale parameters $\left(e_{0} a / L\right)$, power law exponents $(k)$ and different boundary conditions such as S-S and C-C. The bottom surface of the beam is pure metal (SUS304) whereas the top surface of the beam is pure ceramic ( $\mathrm{SiC}$ ). Mechanical properties of nanobeam constituents are given in Table 1. Geometrical properties of the FG nanobeam are: $b$ (width $)=100 \mathrm{~nm}, h$ (thickness) $=200 \mathrm{~nm}$ and $L($ length $)=10000 \mathrm{~nm}$.

Table 1. Properties of FG nanobeams constituents [26]

\begin{tabular}{ccc}
\hline & \multicolumn{2}{c}{ Properties } \\
\cline { 2 - 3 } & E $(\mathbf{G p a})$ & $\boldsymbol{\rho}\left(\mathbf{k g} / \mathbf{m}^{\mathbf{3}}\right)$ \\
\hline Silicon Carbide $(\mathbf{S i C})$ & 427 & 3210 \\
Stainless Steel (SUS304) & 207.78 & 8166 \\
\hline
\end{tabular}

The frequency values obtained from the analyses of S-S FG nanobeam and C-C FG nanobeam with various $e_{0} a / L$ ranging from 0 to 0.5 and various $k$ ranging from 0 to 10 are presented in Table 2 and Table 3 , respectively.

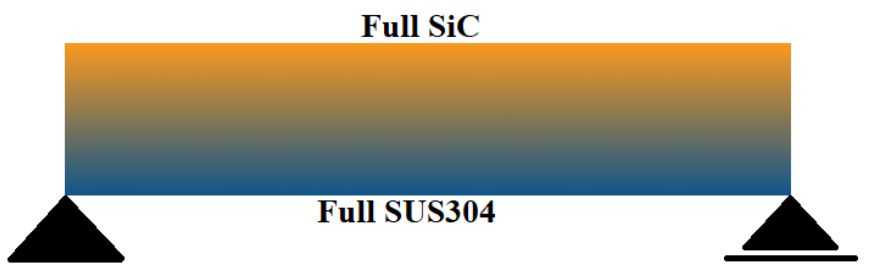

Fig. 3. Functionally graded S-S nanobeam

Table 2. Variation of first five frequencies $(\mathrm{MHz})$ of FG nanobeam with $k$ and $e_{0} a / L(\mathrm{~S}-\mathrm{S})$

\begin{tabular}{|c|c|c|c|c|c|c|c|}
\hline \multirow{2}{*}{$k$} & \multirow{2}{*}{$\begin{array}{c}\omega \\
(\mathbf{M H z})\end{array}$} & \multicolumn{6}{|c|}{ eoa/L } \\
\hline & & $\mathbf{0}$ & 0.1 & 0.2 & 0.3 & 0.4 & 0.5 \\
\hline \multirow[t]{5}{*}{$\mathbf{0}$} & $\omega_{1}$ & 10.4580 & 9.9772 & 8.8551 & 7.6106 & 6.5120 & 5.6163 \\
\hline & $\omega_{2}$ & 41.8114 & 35.4031 & 26.0350 & 19.5949 & 15.4576 & 12.6820 \\
\hline & $\omega 3$ & 93.9986 & 68.4054 & 44.0524 & 31.3427 & 24.1004 & 19.5126 \\
\hline & $\omega_{4}$ & 166.9175 & 103.9357 & 61.7090 & 42.7962 & 32.5689 & 26.2355 \\
\hline & $\omega 5$ & 260.4264 & 139.8566 & 78.9911 & 54.0604 & 40.9330 & 32.8930 \\
\hline \multirow[t]{5}{*}{0.2} & $\omega_{1}$ & 8.8233 & 8.4177 & 7.4710 & 6.4210 & 5.4941 & 4.7384 \\
\hline & $\omega_{2}$ & 35.2750 & 29.8685 & 21.9649 & 16.5316 & 13.0411 & 10.6994 \\
\hline & $\omega 3$ & 79.3007 & 57.7093 & 37.1643 & 26.4418 & 20.3320 & 16.4616 \\
\hline & $\omega_{4}$ & 140.8103 & 87.6793 & 52.0573 & 36.1025 & 27.4749 & 22.1321 \\
\hline & $\omega_{5}$ & 219.6787 & 117.9739 & 66.6317 & 45.6018 & 34.5284 & 27.7464 \\
\hline \multirow[t]{5}{*}{2} & $\omega_{1}$ & 6.1069 & 5.8262 & 5.1709 & 4.4442 & 3.8026 & 3.2796 \\
\hline & $\omega_{2}$ & 24.4163 & 20.6741 & 15.2035 & 11.4427 & 9.0266 & 7.4058 \\
\hline & $\omega_{3}$ & 54.8939 & 39.9478 & 25.7260 & 18.3037 & 14.0743 & 11.3951 \\
\hline & $\omega 4$ & 97.4831 & 60.7005 & 36.0393 & 24.9938 & 19.0209 & 15.3221 \\
\hline & $\omega 5$ & 152.1054 & 81.6851 & 46.1358 & 31.5747 & 23.9074 & 19.2116 \\
\hline 5 & $\omega_{1}$ & 5.5038 & 5.2508 & 4.6602 & 4.0052 & 3.4271 & 2.9557 \\
\hline
\end{tabular}




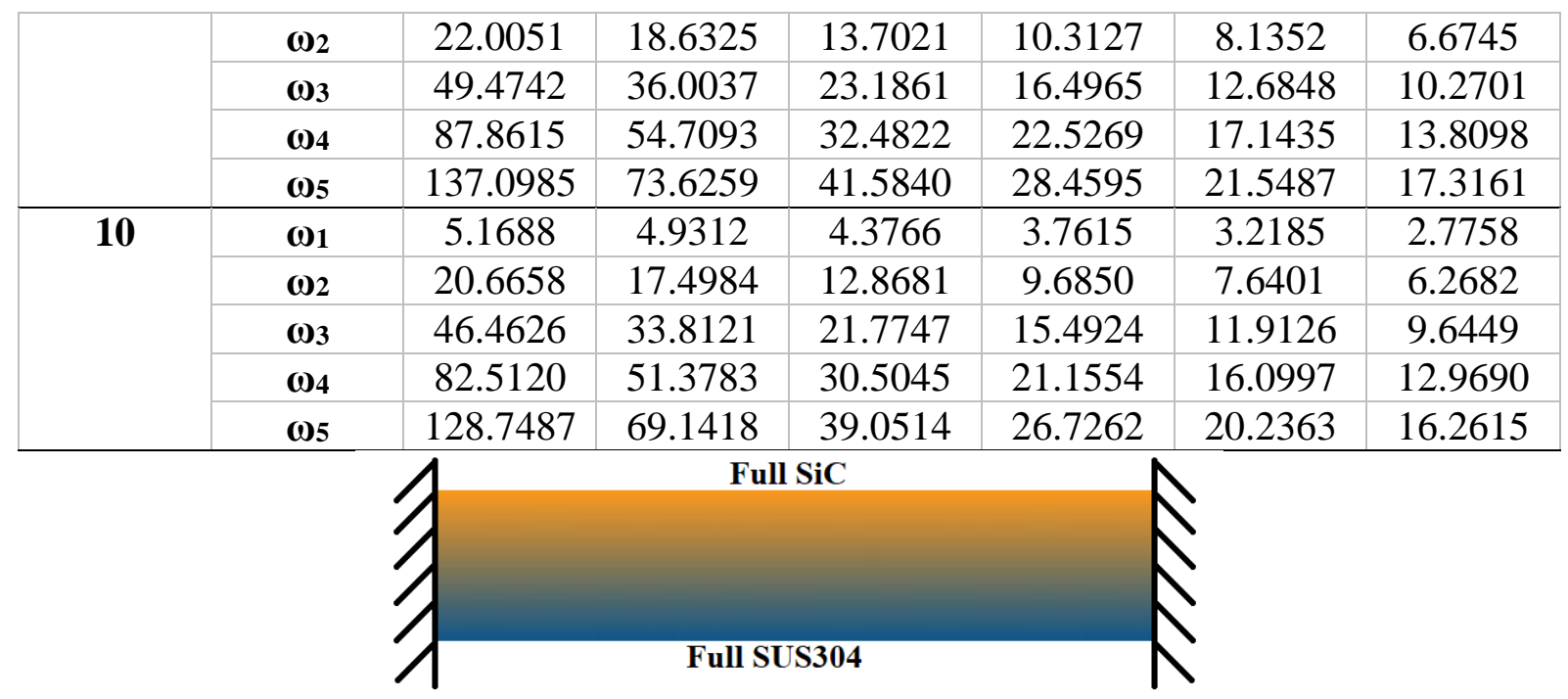

Fig. 4. Functionally graded C-C nanobeam

Table 3. Variation of first five frequencies (MHz) of FG nanobeam with $k$ and $e_{0} a / L(\mathrm{C}-\mathrm{C})$

\begin{tabular}{|c|c|c|c|c|c|c|c|}
\hline \multirow{2}{*}{$k$} & \multirow{2}{*}{$\begin{array}{c}\omega \\
(\mathbf{M H z})\end{array}$} & \multicolumn{6}{|c|}{ eoa/L } \\
\hline & & $\mathbf{0}$ & 0.1 & 0.2 & 0.3 & 0.4 & 0.5 \\
\hline \multirow[t]{5}{*}{$\mathbf{0}$} & $\omega_{1}$ & 23.7062 & 22.3654 & 19.3760 & 16.2643 & 13.6693 & 11.6421 \\
\hline & $\omega_{2}$ & 65.3103 & 53.9796 & 38.5572 & 28.5806 & 22.3751 & 18.2819 \\
\hline & $\omega 3$ & 127.9220 & 90.6560 & 57.6512 & 41.0566 & 31.6753 & 25.7224 \\
\hline & $\omega_{4}$ & 211.2074 & 128.1562 & 75.6097 & 52.4448 & 39.9337 & 32.1811 \\
\hline & $\omega 5$ & 315.0270 & 165.2416 & 93.2586 & 64.0399 & 48.6300 & 39.1599 \\
\hline \multirow[t]{5}{*}{0.2} & $\omega_{1}$ & 20.0006 & 18.8694 & 16.3471 & 13.7218 & 11.5325 & 9.8221 \\
\hline & $\omega_{2}$ & 55.1000 & 45.5403 & 32.5288 & 24.1120 & 18.8767 & 15.4234 \\
\hline & $\omega_{3}$ & 107.9189 & 76.4787 & 48.6349 & 34.6354 & 26.7212 & 21.6993 \\
\hline & $\omega_{4}$ & 178.1710 & 108.1072 & 63.7804 & 44.2395 & 33.6858 & 27.1461 \\
\hline & $\omega_{5}$ & 265.7327 & 139.3795 & 78.6615 & 54.0159 & 41.0180 & 33.0302 \\
\hline \multirow[t]{5}{*}{2} & $\omega_{1}$ & 13.8432 & 13.0603 & 11.3147 & 9.4976 & 7.9823 & 6.7985 \\
\hline & $\omega_{2}$ & 38.1389 & 31.5225 & 22.5165 & 16.6905 & 13.0666 & 10.6763 \\
\hline & $\omega 3$ & 74.7053 & 52.9434 & 33.6689 & 23.9776 & 18.4989 & 15.0223 \\
\hline & $\omega 4$ & 123.3507 & 74.8490 & 44.1601 & 30.6307 & 23.3236 & 18.7956 \\
\hline & $\omega_{5}$ & 183.9983 & 96.5172 & 54.4729 & 37.4063 & 28.4054 & 22.8738 \\
\hline \multirow[t]{5}{*}{5} & $\omega_{1}$ & 12.4760 & 11.7704 & 10.1972 & 8.5597 & 7.1940 & 6.1271 \\
\hline & $\omega_{2}$ & 34.3727 & 28.4098 & 20.2932 & 15.0425 & 11.7765 & 9.6222 \\
\hline & $\omega_{3}$ & 67.3299 & 47.7170 & 30.3455 & 21.6109 & 16.6729 & 13.5395 \\
\hline & $\omega_{4}$ & 111.1767 & 67.4631 & 39.8028 & 27.6084 & 21.0223 & 16.9411 \\
\hline & $\omega_{5}$ & 165.8462 & 86.9977 & 49.1007 & 33.7173 & 25.6041 & 20.6181 \\
\hline \multirow[t]{5}{*}{10} & $\omega_{1}$ & 11.7167 & 11.0541 & 9.5766 & 8.0387 & 6.7562 & 5.7542 \\
\hline & $\omega_{2}$ & 32.2807 & 26.6807 & 19.0580 & 14.1269 & 11.0597 & 9.0365 \\
\hline & $\omega 3$ & 63.2313 & 44.8121 & 28.4980 & 20.2951 & 15.6578 & 12.7152 \\
\hline & $\omega_{4}$ & 104.4073 & 63.3549 & 37.3788 & 25.9271 & 19.7421 & 15.9094 \\
\hline & $\omega 5$ & 155.7450 & 81.6980 & 46.1094 & 31.6632 & 24.0442 & 19.3619 \\
\hline
\end{tabular}




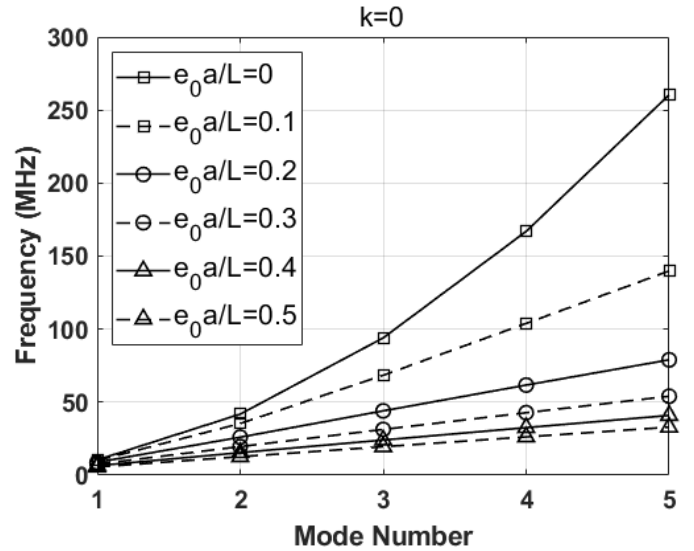

(a)

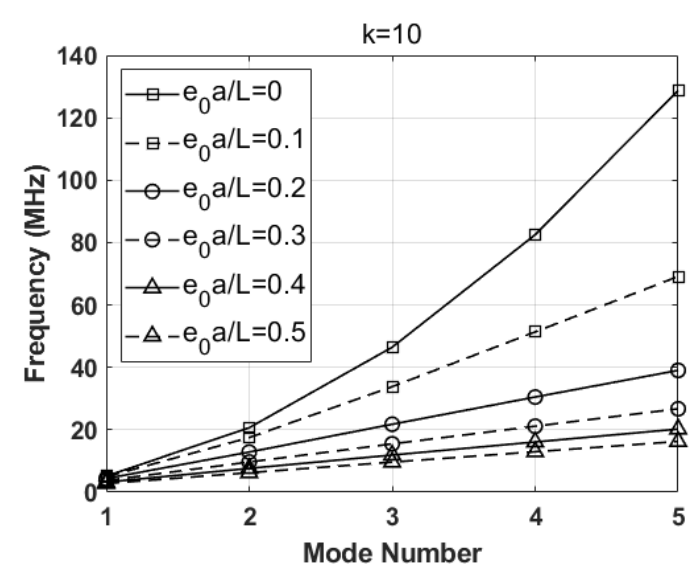

(b)

Fig. 5. The variation of the frequencies with mode numbers for different $e_{0} a / L(\mathrm{~S}-\mathrm{S})$

(a) $k=0$ (b) $k=10$

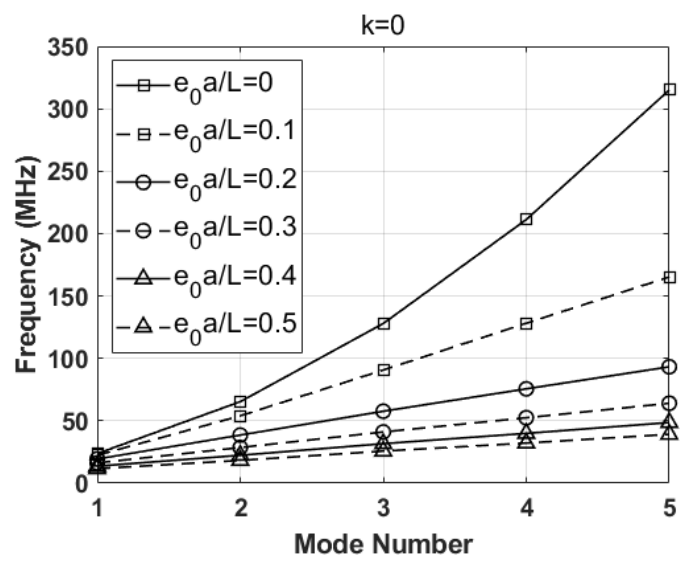

(a)

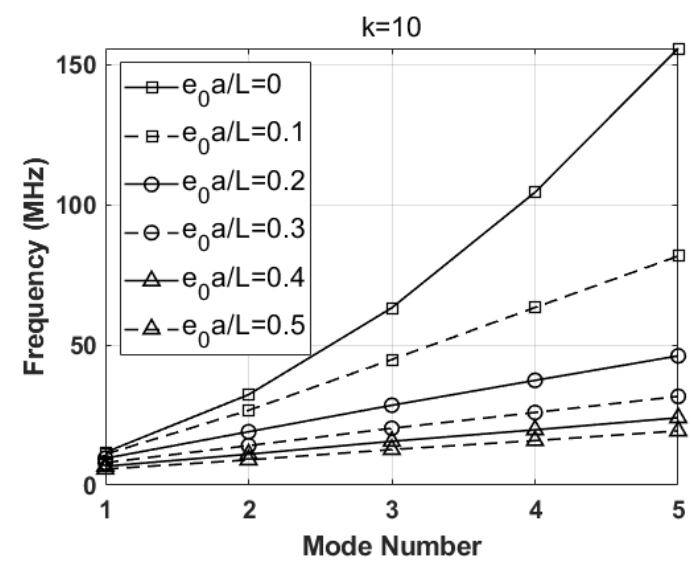

(b)

Fig. 6. The variation of the frequencies with mode numbers for different $e_{0} a / L(\mathrm{C}-\mathrm{C})$ (a) $k=0$ (b) $k=10$

The effects of mode number on the frequency are respectively shown in Fig. 5 and Fig. 6. The frequency values of FG nanobeam increase as the mode number increase. 


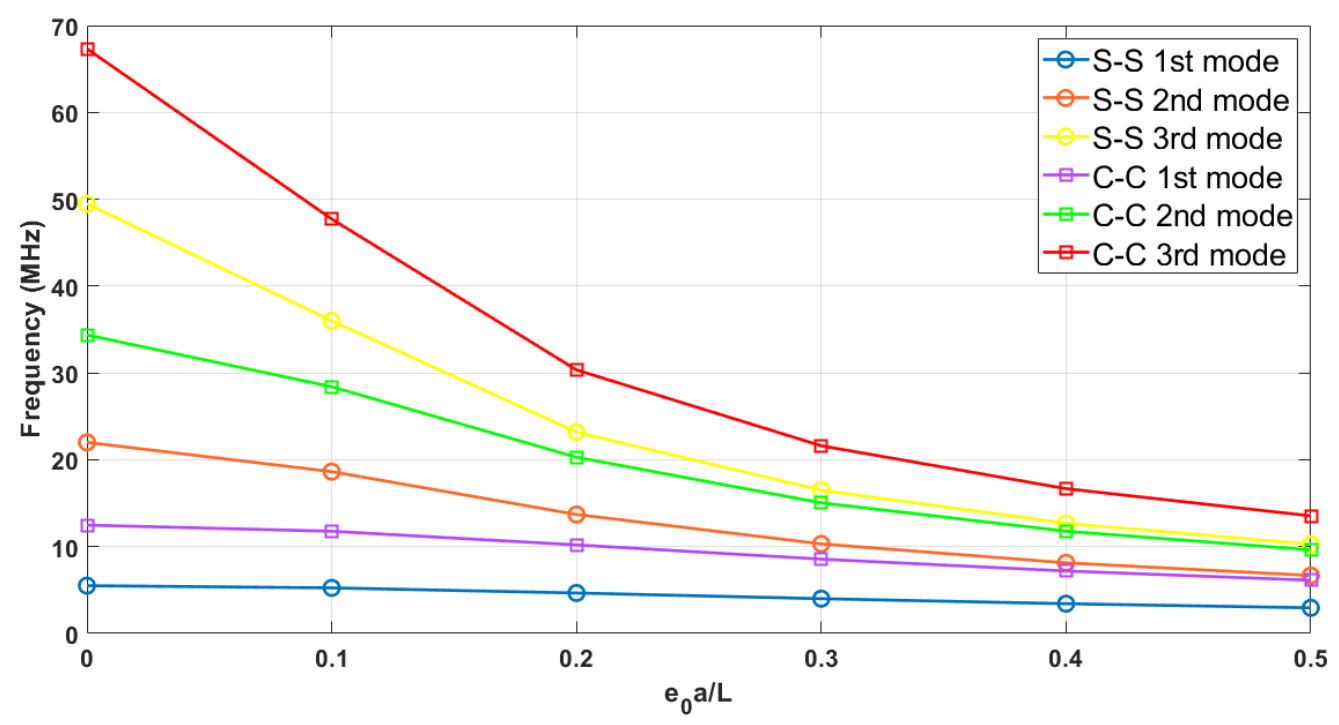

Fig. 7. The variation of the frequencies with $e_{0} a / L(k=5)$

The effects of $e_{0} a / L$ (small-scale parameters) on the frequency are depicted in Fig. 7. The frequency values of FG nanobeam decrease as $e_{0} a / L$ increases.

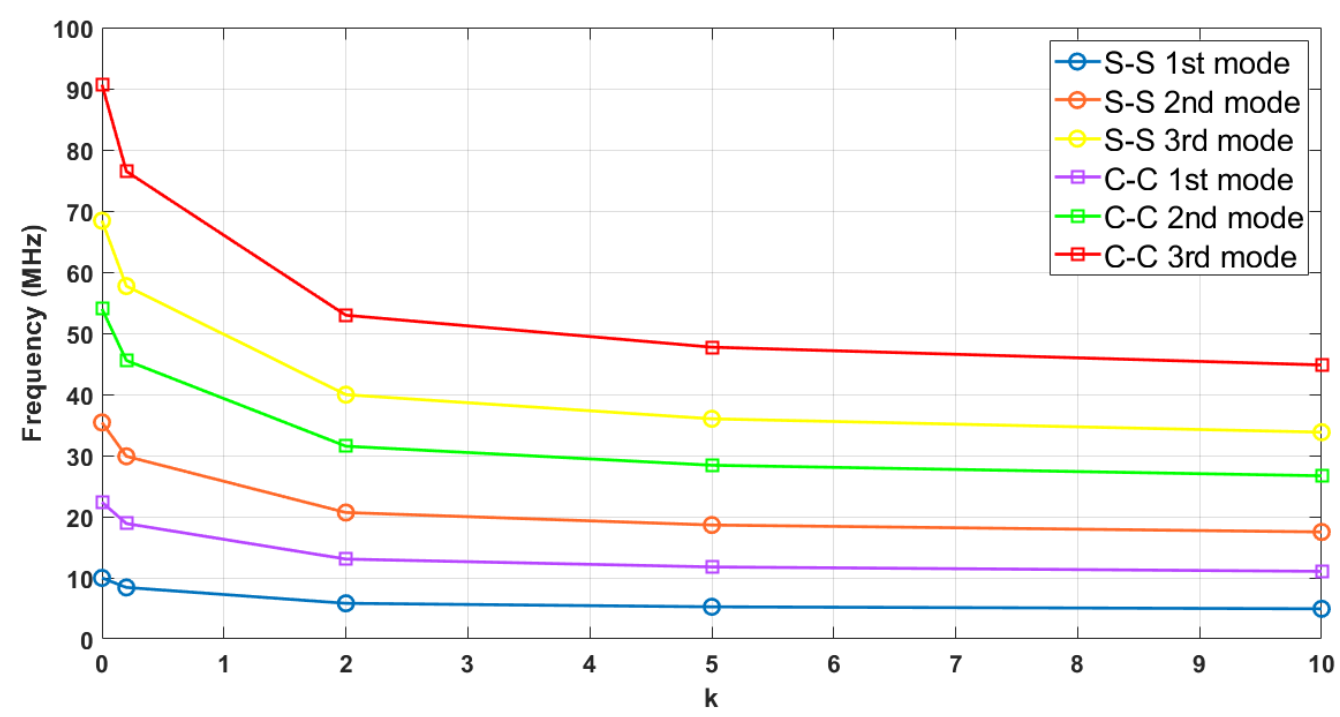

Fig. 8. The variation of the frequencies with $k\left(e_{0} a / L=0.1\right)$

The effects of $k$ (power law exponent) on the frequency are depicted in Fig. 8. The frequency values of FG nanobeam decrease as $k$ increases. Also it is clearly observed from the tables and figures that the frequency values of $\mathrm{C}-\mathrm{C}$ boundary condition higher than the frequency values of S-S boundary condition. 


\section{Conclusions}

Due to the small-scale effect, the properties and behaviours of nano structures are different from macro structures. In this paper, free vibration analysis of FG nanobeam composed of $\mathrm{SiC}$ and SUS304 is investigated based on the nonlocal elasticity theory and Euler-Bernoulli beam theory. Finite element method is a powerful numerical method. A nonlocal finite element formulation is developed for free vibration analysis of FG nanobeams, in this study. Solutions are obtained for S-S and C-C FG nanobeams. According to the obtained results

- By increasing $e_{0} a / L$, the frequency values decrease.

- Frequencies decrease with increasing $k$ value.

- The frequency values of S-S smaller than the frequency values of C-C.

- The frequency values increase as the mode number increase.

- As the $k$ increases, the properties of the FG nanobeam transform from ceramic to metal.

\section{References}

[1] Akgöz, B. and Civalek, Ö., Free vibration analysis of axially functionally graded tapered Bernoulli-Euler microbeams based on the modified couple stress theory. Composite Structures, 98, 314-322, 2013.

[2] Ebrahimi, F., Ghadiri, M., Salari, E., Hoseini, S.A.H. and Shaghaghi, G.R., Application of the differential transformation method for nonlocal vibration analysis of functionally graded nanobeams. Journal of Mechanical Science and Technology, 29(3), 1207-1215, 2015.

[3] Yayli, M.Ö. Torsional vibrations of restrained nanotubes using modified couple stress theory. Microsystem Technologies, 1-11, 2018.

[4] Ansari, R., Gholami, R., and Sahmani, S., Free vibration analysis of size-dependent functionally graded microbeams based on the strain gradient Timoshenko beam theory. Composite Structures, 94(1), 221-228, 2011.

[5] Akgöz, B., and Civalek, Ö., Effects of thermal and shear deformation on vibration response of functionally graded thick composite microbeams. Composites Part B: Engineering, 129, 77-87, 2017.

[6] Eringen, A.C., On differential equations of nonlocal elasticity and solutions of screw dislocation and surface waves. Journal of applied physics, 54(9), 4703-4710, 1983.

[7] Naghinejad, M. and Ovesy, H.R., Free vibration characteristics of nanoscaled beams based on nonlocal integral elasticity theory. Journal of Vibration and Control, 24(17), 39743988, 2018. 
[8] Yayl1, M.Ö., Buckling Analysis of a Rotationally Restrained Single Walled Carbon Nanotube Embedded In An Elastic Medium Using Nonlocal Elasticity. International Journal Of Engineering \& Applied Sciences, 8(2), 40-50, 2016.

[9] Eltaher, M.A., Emam, S.A. and Mahmoud, F.F., Static and stability analysis of nonlocal functionally graded nanobeams. Composite Structures, 96, 82-88, 2013.

[10] Thai, H.T., A nonlocal beam theory for bending, buckling, and vibration of nanobeams. International Journal of Engineering Science, 52, 56-64, 2012.

[11] Tounsi, A., Benguediab, S., Adda, B., Semmah, A. and Zidour, M., Nonlocal effects on thermal buckling properties of double-walled carbon nanotubes. Advances in nano research, 1(1), 1-11, 2013.

[12] Thai, S., Thai, H.T., Vo, T.P. and Patel, V.I., A simple shear deformation theory for nonlocal beams. Composite Structures, 183, 262-270, 2018.

[13] Ebrahimi, F. and Salari, E., Thermo-mechanical vibration analysis of nonlocal temperature-dependent FG nanobeams with various boundary conditions. Composites Part B: Engineering, 78, 272-290, 2015.

[14] Yayli, M.Ö., Buckling analysis of a cantilever single-walled carbon nanotube embedded in an elastic medium with an attached spring. Micro \& Nano Letters, 12(4), 255-259, 2017.

[15] Yayli, M.Ö., On the axial vibration of carbon nanotubes with different boundary conditions. Micro \& Nano Letters, 9(11), 807-811, 2014.

[16] Civalek, Ö., and Demir, C, Buckling and bending analyses of cantilever carbon nanotubes using the euler-bernoulli beam theory based on non-local continuum model. Asian Journal of Civil Engineering, 12(5), 651-661, 2011.

[17] Demir, Ç., and Civalek, Ö., A new nonlocal FEM via Hermitian cubic shape functions for thermal vibration of nano beams surrounded by an elastic matrix. Composite Structures, 168, 872-884, 2017.

[18] Naghinejad, M., and Ovesy, H.R., Viscoelastic free vibration behavior of nano-scaled beams via finite element nonlocal integral elasticity approach. Journal of Vibration and Control, 25(2), 445-459, 2019.

[19] Yayli, M.Ö., Effects of rotational restraints on the thermal buckling of carbon nanotube. Micro \& Nano Letters, 14(2), 158-162, 2019.

[20] Mercan, K., Numanoglu, H.M., Akgöz, B., Demir, C. and Civalek, Ö., Higher-order continuum theories for buckling response of silicon carbide nanowires (SiCNWs) on elastic matrix. Archive of Applied Mechanics, 87(11), 1797-1814, 2017.

[21] Wang, C.M., Zhang, Y. Y., Ramesh, S.S. and Kitipornchai, S., Buckling analysis of micro-and nano-rods/tubes based on nonlocal Timoshenko beam theory. Journal of Physics D: Applied Physics, 39(17), 3904, 2006. 
[22] Civalek, Ö. and Demir, Ç., Bending analysis of microtubules using nonlocal EulerBernoulli beam theory. Applied Mathematical Modelling, 35(5), 2053-2067, 2011.

[23] Kadıoğlu, H.G. and Yaylı, M.Ö., Buckling Analysis of Non-Local Timoshenko Beams by Using Fourier Series. International Journal Of Engineering \& Applied Sciences, 9(4), 89-99, 2017.

[24] Zargaripoor, A., Daneshmehr, A., Isaac Hosseini, I., and Rajabpoor, A., Free vibration analysis of nanoplates made of functionally graded materials based on nonlocal elasticity theory using finite element method. Journal of Computational Applied Mechanics, 49(1), 86-101, 2018.

[25] Reddy, J.N., Energy Principles and Variational Methods in Applied Mechanics, John Wiley \& Sons; 2nd edition, 2002.

[26] Talha, M. and Singh, B.N., Static response and free vibration analysis of FGM plates using higher order shear deformation theory. Applied Mathematical Modelling, 34(12), 3991-4011, 2010. 